\title{
Ionization of large-scale absorbing haloes and feedback events from high-redshift radio galaxies
}

\author{
L. Binette ${ }^{1}$, R. J. Wilman ${ }^{2}$, M. Villar-Martín ${ }^{3}$, R. A. E. Fosbury ${ }^{4}$, M. J. Jarvis ${ }^{5}$, and H. J. A. Röttgering ${ }^{6}$ \\ 1 Instituto de Astronomía, UNAM, Ap. 70-264, 04510 México, DF, México \\ 2 Department of Physics, University of Durham DH1 3LE, UK \\ e-mail: r.j.wilman@durham.ac.uk \\ 3 Instituto de Astrofísica de Andalucía, CSIC, Apdo. 3004, 18080 Granada, Spain \\ 4 ST-ECF, Karl-Schwarzschild Strasse 2, 85748 Garching bei München, Germany \\ 5 Astrophysics Department, Keble Road, Oxford OX1 3RH, UK \\ ${ }^{6}$ Leiden Observatory, PO Box 9513, 2300 RA, Leiden, The Netherlands
}

Received 24 February 2006 / Accepted 7 July 2006

ABSTRACT

\begin{abstract}
Aims. We present photoionization calculations for the spatially-extended absorbers observed in front of the extended emission-line spectrum of two high-redshift radio galaxies, 0943-242 $\left(z_{\mathrm{e}}=2.922\right)$ and $0200+015\left(z_{\mathrm{e}}=2.230\right)$, with the aim of reproducing the absorber column ratio, $N_{\mathrm{CIV}} / N_{\mathrm{HI}}$.

Methods. We explore the effects of using different UV continua in the photoionization calculations. A comparison is made between the absorber in 0200+015 and the two absorbers observed near the lensed Lynx arc nebula at redshift 3.36, which present very similar $N_{\text {CIV }} / N_{\text {HI }}$ ratios.

Results. We find that hot stars from a powerful starburst, or a metagalactic background radiation (MBR) in which stars dominate quasars, are equally successful in reproducing the observed $N_{\mathrm{CIV}} / N_{\mathrm{HI}}$, assuming subsolar gas metallicities for each absorber. These softer SEDs eliminate the difference of a factor 1000 in metallicity between the two absorbers encountered in earlier work where a power-law SED was assumed. The detection of continuum flux in 0943-242 suggests that the level of ionizing photons is consistent with a stellar ionizing source.

Conclusions. If the MBR is responsible for the ionization of the radio galaxy absorbing shells, their radii (if spherical) would be large $(>100 \mathrm{kpc})$ and their mass huge $>10^{12} M_{\odot}$, implying that the feedback mechanism initiated by the central galaxy has caused the expulsion of more baryonic mass than that left in the radio galaxy. If, as we believe is more likely, stellar ionizing sources within the radio galaxy are responsible for the absorber's ionization, smaller radii of $\sim 25 \mathrm{kpc}$ and much smaller masses $\left(\sim 10^{8}-10^{10} M_{\odot}\right)$ are inferred. This radius is consistent with the observed transition in radio source size between the smaller sources in which strong $\mathrm{HI}$ absorption is almost ubiquitous and the larger sources where it is mostly lacking. Finally, we outline further absorption-line diagnostics that could be used to further constrain the properties of the haloes and their source of ionization.
\end{abstract}

Key words. cosmology: early universe - galaxies: active - galaxies: formation - galaxies: ISM - line: formation

\section{Introduction}

A prominent characteristic of high-redshift radio galaxies (HzRGs) at $z>2$ is their spatially extended line emission regions (hereafter EELR), which are often luminous in Ly $\alpha$ $\left(>10^{44} \mathrm{erg} \mathrm{s}^{-1}\right)$ and extended over several to tens of $\mathrm{kpc}$. The excitation mechanism for the emission gas is either shock excitation by jet material or AGN photoionization (the presence of $\mathrm{N}$ V $\lambda 1240$ line emission precludes stellar photoionization). The EELR is kinematically active, with FWHM reaching $1000 \mathrm{~km} \mathrm{~s}^{-1}$. With observations of a sample of HzRGs, Van Ojik et al. (1997, VO97) discovered that, when observed at intermediate resolution (1-2 $\AA$ ), the majority of HzRGs with small radio-source sizes $(<50 \mathrm{kpc})$ exhibit narrow Ly $\alpha \mathrm{HI}$ absorption. This absorption is superimposed upon the Ly $\alpha$ emission with a spatial extent comparable to that of the EELR. In addition to Ly $\alpha$, the C IV $\lambda \lambda 1549$ doublet has also been observed in absorption in two HzRGs, superimposed on the CIV emission line, first in 0943-242 $\left(z_{\mathrm{e}}=2.922\right)$ (Binette et al. 2000, hereafter B00) and second in $0200+015\left(z_{\mathrm{e}}=2.230\right)$ (Jarvis et al. 2003, hereafter J03). Building on the results of B00 and J03 in the present paper, we examine the excitation mechanism of the large-scale absorbing haloes in greater detail by exploring photoionization with a variety of different spectral energy distributions (hereafter SED).

The basic structure of the paper is as follows. In the remainder of Sect. 1 we review our current understanding of HzRG absorbers, focussing on the distribution, ionization, and metallicity of the absorbing gas and on the specific problems that motivate our current study; an insightful comparison is made with the absorbers in the Lynx arc nebula (LAN), a gravitationally-lensed H II galaxy at $z=3.357$. In Sect. 2 we summarise the observational results we aim to reproduce, namely the $N_{\mathrm{CIV}} / N_{\mathrm{HI}}$ ratio in the aforementioned HzRGs and the LAN. Section 3 describes the MAPPINGS Ic code and our assumptions concerning the photoionizing SEDs. Section 4 presents the results of these calculations and in Sect. 5 we assess their implications for the origins of the absorbers and their compatibility with other observables. Finally, in Sect. 6 we present some additional absorption-line diagnostics that may help in the future to distinguish between the proposed scenarios. 


\subsection{Shell-like structure for the $\mathrm{HzRG}$ haloes}

Among the HzRGs with small radio sources $(<50 \mathrm{kpc})$, the detection rate of associated absorption systems is $90 \%$ (9 out of $10 \mathrm{HzRGs}$ in the V097 study), while it is only $25 \%$ for larger radiosizes. The fact that the absorption extends over the whole background EELR emission favours a shell-like geometry for the absorption systems rather than a conglomerate of individual clouds, as proposed initially by VO97. In Sect. 1.3 we give further indications as to why we retain the simplifying assumption of a simple shell structure in the current work. Because the density-per-unit redshift of the strong absorbers $\left(N_{\mathrm{HI}}>10^{18} \mathrm{~cm}^{-2}\right)$ around HzRGs was found to be much higher than that given by the statistics of intergalactic medium (IGM) absorbers at large, VO97 inferred that they belong to the environment of the parent HzRG rather than to the IGM. The density of the thinnest absorbers $\left(<10^{15} \mathrm{~cm}^{-2}\right)$ around $\mathrm{H} z \mathrm{RGs}$, on the other hand, is comparable to that of $\operatorname{Ly} \alpha$ forest absorbers in the IGM, as more recently shown by Wilman et al. (2004: W04). It is conceivable that the physical conditions in the thin ${ }^{1} \mathrm{H} z \mathrm{RG}$ absorbers are indistinguishable from those operating within typical IGM Ly $\alpha$ forest absorbers. The available data, however, are still insufficient to confirm or refute this proposition.

The rarity of absorbers among HzRGs with radiosizes larger ${ }^{2}$ than $50 \mathrm{kpc}$ suggests that the typical lateral dimensions of the shell (in the plane of the sky) might be $\$ 50 \mathrm{kpc}$. The proposed interpretation is that, as the AGN jet expands beyond this size, the bow-shocks overtake the shells and disrupt them. This is the first scenario, which we label A or the "inner shell scenario". If valid, it suggests that the expansion of the AGN jet cocoon is not the mechanism by which the shells are formed, but rather by which they are destroyed. Scenario A favours a shell-formation mechanism that relies on large-scale outflows generated by episodes of massive star formation. Using highdispersion data from VLT-UVES, W04 propose that the absorbers in HzRGs probably lie within the core of young galactic protoclusters, consistent with observations of their environments (e.g. Venemans et al. 2005; Overzier et al. 2006) and may be a byproduct of massive galaxy formation. Krause (2005) published hydrodynamical simulations of the formation of a shell due to the expansion of a stellar-wind bowshock. At a later stage in his model, an AGN jet is launched and a jet cocoon builds up. Once the jet has extended beyond the initial bow-shock, the jet cocoon destroys the shell as it overtakes it. An estimate of the timescale for this to occur can be obtained if one follows the reasoning of $\mathrm{J} 03$, where the radiosize represents a kind of internal clock (see Sect. 1.5), which characterises not only the radio jet's age but also that of the starburst superwind that generates the shells.

A second possibility is that the rarity of shells among HzRGs with large radiosizes may reflect an older phase in which the shells have expanded farther out and thinned out considerably. This process would eventually render them undetectable (using the VO97 detection technique) when their $N_{\mathrm{HI}}$ columns drop below $\$ 10^{13} \mathrm{~cm}^{-2}$. This is the second scenario, which we label B or the "aging shell scenario". In this case, the distance between the shell and the parent HzRG is unknown and can be much larger than the upper-limit size implied by scenario A, as will be discussed in Sect. 5.1. Scenario B leaves the possibility open that

\footnotetext{
${ }^{1}$ By "thin" we refer to an H I column density, not to a small physical size.

${ }^{2}$ VO97 (p. 369) showed that this finding is not the result of a selection effect due to the fact that larger radio sources have a narrower Ly $\alpha$ emission profile.
}

some of the shells may result from the expansion of a jet bowshock (e.g. Krause 2002), although the most likely formation mechanism of the shells remains a stellar superwind, as in scenario A.

The large-scale $\mathrm{H} z \mathrm{RG}$ absorbers might be analogous to the absorbers detected within ${ }^{3} 20-50 h^{-1} \mathrm{kpc}$ of high redshift galaxies by Adelberger et al. (2005) using nearby-field spectroscopy of background QSOs or galaxies. The advantage of HzRG absorber studies is that the intrinsic shell outflow velocity is more readily available from observations, but not their distance from the parent HzRG (the reverse applies to the technique used by Adelberger et al., assuming spherical expansion of the absorbers).

The HzRG shells share many similarities with at least some of Ly $\alpha$-emitting "blobs" (hereafter LAB; Steidel et al. 2000) associated with Lyman break galaxies (Pettini et al. 2001), that are characterised by an EELR that can reach large sizes of up to $\sim 100 \mathrm{kpc}$. An important difference is that the radio luminosities are much fainter or even undetected in the latter case. Using integral field spectroscopy, Wilman et al. (2005) observed the Ly $\alpha$ emitting "blob" LAB-2 in the SSA22 protocluster at $z_{\mathrm{e}}=3.09$, and discovered a foreground absorber $\left(N_{\mathrm{HI}} \simeq 10^{19} \mathrm{~cm}^{-2}\right)$ with remarkable velocity coherence over a projected size of $\sim 76 \times$ $26 \mathrm{kpc}$. Their interpretation is that a galaxy-wide superwind swept up $\sim 10^{11} M_{\odot}$ of diffuse material from the IGM over a few $10^{8}$ yr. This is a manifestation of the "feedback" mechanism thought to be regulating the formation of galaxies.

\subsection{What is ionizing the large-scale haloes?}

At least a fraction of the known haloes appear to be highly ionized. In both HzRGs in which a C IV $\lambda \lambda 1549$ doublet has been observed in absorption (0943-242 and 0200+015), the absorption redshift corresponds to one of the Ly $\alpha$ absorbers. These two absorbing haloes therefore contain ionization species up to at least $\mathrm{C}^{+3}$. B00 and J03 assume that the H I and C IV absorption species occur within a physically contiguous structure, an aspect discussed further in Sect. 1.3.

The possibility that the HzRG absorbing haloes are photoionized by the hidden nuclear radiation can be ruled out. First, because there no observed continuum of sufficient strength underlying the EELR. This is as expected in the quasar-radio galaxy unification picture (Barthel 1989; Antonucci 1993; Haas et al. 2005), in which the nuclear ionizing radiation is collimated along two ionization cones, which in radio galaxies lie along the plane of the sky, and is therefore invisible to the observer and presumably also to the intervening absorbers, unless rather contrived gas geometries are postulated. Second, B00 shows that the C IV/Ly $\alpha$ emission- and absorption-line ratios in 0943-242 could not be reconciled with any model in which the absorptionand emission gases are co-spatial. They concluded that the absorbing gas has much lower metallicity and is located farther away from the host galaxy than the EELR. Although they favoured the idea that the diffuse metagalactic background radiation (hereafter MBR) rather than the parent AGN was responsible for ionizing the absorbing haloes, in their calculations B00 and J03 used a simple power law as a crude approximation of the MBR energy distribution. In this paper, we assume more realistic SEDs that take the cumulative opacity of IGM Lyman limit systems and Ly $\alpha$ forest absorbers into account.

As for the possibility of any collisional ionization of the shells, we indicated in J03 that this mechanism was unlikely in

\footnotetext{
${ }^{3}$ Where $h=H_{0} / 100 \mathrm{~km} \mathrm{~s}^{-1} \mathrm{Mpc}^{-1}$.
} 
the case of $0200+015$ and that steady-state photoionizing shocks (Dopita \& Sutherland 1996) resulted in rather large $N_{\mathrm{HI}}$ columns $\left(\sim 10^{19} \mathrm{~cm}^{-2}\right)$, incompatible with the low value characterising $0200+015$. In the case of 0943-242, the near-solar metallicity models of Dopita \& Sutherland (1996) do not attain the observed $N_{\text {CIV }}$ value for shock velocities below $400 \mathrm{~km} \mathrm{~s}^{-1}$ and, above this velocity, the $N_{\mathrm{HI}}$ column becomes excessive, requiring the shock structure to be truncated. As for the photoionized precursor nebula upstream from the shocks, the SED generated downstream by fast shocks is as hard as a power law of index $\alpha \simeq-0.5$ up to $\gtrsim 500 \mathrm{eV}$ (Binette et al. 1985). Therefore, photoionization calculations with a power law as presented in Sect. 4.2 capture the main features of such a precursor. In essence, any hard SED requires supersolar metallicities in order to fit the column ratios found in $0200+015$. Finally, calculations to represent the case of a collisionally ionized gas slab at temperature $T$ has been explored by J03. They find that for $0200+015$, the $N_{\mathrm{CIV}} / N_{\mathrm{HI}}$ column ratio could be reproduced by using roughly solar metallicities, provided that $T$ is finetuned to lie around $10^{5} \mathrm{~K}$. Apart from the fact that this metallicity is rather high for the redshift considered, it would be difficult to explain how the plasma could be maintained at a temperature approaching the peak of its cooling curve. This would most likely require a yet unknown heating mechanism.

\subsection{The case for a simple scattering screen}

In a morphologically and kinematically complex EELR, one cannot readily disentangle photon destruction due to line-of-sight absorption from the effects of transmission by multiple scatterings. Nevertheless, for the large-scale absorbing haloes in 0200+015 and 0943-242 (or other HzRGs studied by VO97), there is no evidence that the absorbers share the complexity of the EELR. These results suggest that a uniform foreground scattering screen provides an adequate description of the "absorbing" haloes in HzRGs. Strong evidence of this was provided by observations at much higher spectral resolution using the VLTUVES (e.g. J03 and W04). In particular, J03 finds that the main absorber in 0943-242 remains as a single system of column density $\sim 10^{19} \mathrm{~cm}^{-2}$ over the full size of the EELR, being completely black at its base, with no evidence of a substructure or a multiphase environment. The absorption trough is blueshifted by $265 \mathrm{~km} \mathrm{~s}^{-1}$ with respect to the centroid of the backgroundemission profile. This spatial and kinematical coherence of the absorber contrasts with the chaotic multiphase medium encountered in the Galactic ISM or the EELR of HzRGs. It also suggests that the absorber is physically separate from the background EELR and that it is therefore simply acting as a scattering surface, as argued in J03. This clean separation between EELR and the absorber simplifies the modelling task and justifies the ionizationstratified slab approximation adopted in Sect. 4.

\subsection{Comparison with the Lynx arc nebula}

Following an independent study of the lensed Lynx arc nebula $^{4}$ (LAN) at $z=3.357$ by some of us (Fosbury et al. 2003), it was observed that the column ratios $N_{\mathrm{CIV}} / N_{\mathrm{HI}}$ in the LAN and $0200+015$ are very similar. In the calculations that follow, we therefore explicitly compare the LAN and the HzRGs absorbers, making use of the following insights that place the physical

${ }_{4}$ The Lynx arc nebula is a high-redshift, metal-poor, gravitationallylensed H II galaxy that was discovered serendipitously by Holden et al. (2001). conditions in the LAN on a firm footing. First, the LAN is an active star-forming object, so we may reasonably assume that the subsolar metallicity that characterises the emission gas, $\sim 10 \%$ (following the work of VM04), also applies to the absorbing gas. Second, the LAN presents a relatively high-excitation emission line UV spectrum, which photoionization by hot stars can reproduce successfully. Photoionization by a straight power law ${ }^{5}$, on the other hand, would result in the emission of a detectable $\mathrm{N}$ V $\lambda 1240$ line (comparable in strength to N IV] $\lambda 1485$ line, see BG03), which is not observed. Hence, we know the absorber metallicity and excitation source for this object with some confidence. Therefore, the successful reproduction of the LAN column ratios in Sect. 4.4 using subsolar metallicities and photoionization by hot stars, prompts us to consider that such an SED might also apply to the $\mathrm{HzRG}$ absorbers.

\subsection{Metallicity evolution vs. softer ionizing SED}

With the VLT-UVES, J03 obtained superb spectra of the aforementioned HzRGs at ten times the resolution used by VO97. The spectra confirmed that the main absorber in 0943-242 exhibits no additional substructure to that reported by VO97, as already discussed. In contrast, a very different view of $0200+015$ emerges: the single absorber with HI column density $\sim 10^{19} \mathrm{~cm}^{-2}$ seen at low resolution now splits into two $\sim 10^{14.6} \mathrm{~cm}^{-2}$ systems; these extend by more than $15 \mathrm{kpc}$ to obscure additional Ly $\alpha$ emission coincident with a radio lobe. Additional but fragmented absorbers are seen on the red wing of the emission line at this position. We recall that gas metallicities as high as $\sim 10 Z_{\odot}$ are required to reproduce the $N_{\mathrm{CIV}} / N_{\mathrm{HI}}$ ratio in $0200+015$ (Sect. 1.5; J03) assuming photoionization by a straight power law. This suggests that the absorbing gas has undergone very substantial metal enrichment. Based on the smaller radio source size in 0943-242 (26 kpc versus $43 \mathrm{kpc}$ for $0200+015$ ), J03 conjectured that the radio source age (as inferred from its linear size) is the parameter controlling the evolution of (i) the structure/kinematics of the absorbing halo, through interaction and shredding of the initially quiescent shells, and of (ii) its metallicity build-up, through enrichment by the starburst superwind triggered concurrently with the nuclear radio source.

Although this age and enrichment scenario (B) remains an appealing possibility, the large metallicity gap inferred by $\mathrm{J} 03$ of three orders of magnitude between 0943-242 $\left(\sim 0.01 Z_{\odot}\right)$ and $0200+015\left(\sim 10 Z_{\odot}\right)$ is a cause for concern. Here we revisit the issue by exploring alternative ionizing SEDs that would require only a factor of ten metallicity enhancement with respect to 0943-242. We focus on the case of SEDs from hot stars and the diffuse MBR with the aim of generating a grid of models for comparision with future observations.

\section{The observational dataset}

In this section, we gather together the principal observational results that we aim to reproduce, namely the $\mathrm{H} \mathrm{I}$ and $\mathrm{C}$ IV column densities for the absorbers in the two HzRGs and the LAN.

The C IV and the Ly $\alpha$ absorption columns in 0943-242 $\left(z_{\mathrm{e}}=\right.$ $2.922)$ and $0200+015\left(z_{\mathrm{e}}=2.230\right)$ have been measured by various authors (Röttgering et al. 1995; B00, J03, W04). We adopt

\footnotetext{
5 The possibility that the LAN corresponded to photoionization by a quasar power-law that is partly absorbed was investigated by Binette et al. (2003: BG03). In order to work, such an absorbed power-law model requires a very specific and unlikely fine tuning of the parameters defining the EELR and the hidden filtering screen.
} 
the values of J03, which are based on VLT-UVES observations of both HzRGs. In 0943-242 the dominant large-scale absorber is characterised by an $N_{\mathrm{HI}}$ column of $10^{19.1} \mathrm{~cm}^{-2}$, which puts it among the group of larger H I columns (see W04). However, the four $\operatorname{Ly} \alpha$ absorbers observed in $0200+015$ are rather thin, with columns of the order of $10^{14.7} \mathrm{~cm}^{-2}$. These all belong to the group of smaller H I columns haloes, which are much more numerous (see W04). In 0943-242, the C IV $\lambda \lambda 1548,1551$ doublet is observed in absorption at the same redshift as the dominant H I absorber (J03; B00; Röttgering \& Miley 1997) and corresponds to a column of $10^{14.6} \mathrm{~cm}^{-2}$. In the case of $0200+015$, only one HI absorber with $N_{\mathrm{HI}}=10^{14.7} \mathrm{~cm}^{-2}$ shows a corresponding C IV doublet in absorption, with $N_{\mathrm{CIV}}=10^{14.6} \mathrm{~cm}^{-2}$. The $N_{\mathrm{CIV}} / N_{\mathrm{HI}}$ column ratios for $0943-242$ and $0200+015$ are $10^{-4.5}$ and $10^{-0.07}$, respectively.

As for the LAN, the two local absorption systems have been labelled $a 1$ and $a 2$ by Fosbury et al. (2003) who determined the $\mathrm{H}$ I column to be $1.05 \times 10^{15}$ and $0.60 \times 10^{15} \mathrm{~cm}^{-2}$, respectively, and the C IV columns to be $0.83 \times 10^{15}$ and $1.02 \times 10^{15} \mathrm{~cm}^{-2}$. The $N_{\mathrm{CIV}} / N_{\mathrm{HI}}$ column ratios for $a 1$ and $a 2$ are therefore $10^{-0.10}$ and $10^{0.23}$, respectively. The similarity of $a 1$ to $0200+015$ is noteworthy.

\section{Photoionization models and ionizing energy distributions}

To compute the $N_{\mathrm{CIV}} / N_{\mathrm{HI}}$ ratio, we have used the code MAPPINGS Ic (Binette et al. 1985; Ferruit et al. 1997). To represent solar abundances, we adopted the set of Anders \& Grevesse (1989). When varying metallicities, we multiplied the solar abundances of all elements heavier than He by a constant, which we labelled the gas metallicity (in units of $Z_{\odot}$ ). For the HzRG absorber, we assumed a slab geometry illuminated on one side. For each ionizing SED that we considered, we calculated the equilibrium ionization state of the gas and integrated the ionization structure inward until a preselected target value of the column $N_{\mathrm{HI}}$ was reached. For the range of parameters explored in this paper - where the aim was to reproduce the observed $N_{\mathrm{HI}}$ and the $N_{\text {CIV }} / N_{\text {HI }}$ column ratios - all models of the absorbers turn out to be matter-bounded $(0200+015)$ or marginally optically thick to the ionizing radiation (0943-242). We now describe the different SEDs used in the calculations.

\subsection{Continuum softness}

For a given input SED, the calculations were repeated for different values of the ionization parameter ${ }^{6}$ in order to build a sequence of models in $U$, starting at the minimum value of 0.001 .

It is customary to define the SED's softness using the parameter $\eta$, which is the column ratio of singly ionized $\mathrm{He}$ to neutral $\mathrm{H}$, $N_{\text {HeII }} / N_{\text {HI }}$. This ratio does not, however, uniquely define the SED, as $\eta$ also depends on the slab thickness and on $U$ and not just on the continuum's shape (see for instance Appendix A of Fardal et al. 1998). In the case of stellar SEDs, $\eta$ varies rather abruptly with $T_{\text {eff. }}$ For instance, $\eta$ is 1480 for a $71000 \mathrm{~K}$ star, while its value is only 95 for a $80000 \mathrm{~K}$ star. Next to each SED in Fig. 1, we indicate the value of $\eta$ calculated between brackets, assuming $N_{\mathrm{HI}}=10^{14.8} \mathrm{~cm}^{-2}$ and $U=0.1$. We now review the various SEDs displayed in Fig. 1 and used in the calculations reported in Sect. 4.

6 We use the customary definition of the ionization parameter $U=$ $\varphi_{\mathrm{H}} / c n_{\mathrm{H}}$ as the ratio between the density of ionizing photons impinging on the slab $\varphi_{\mathrm{H}} / c$ and the total $\mathrm{H}$ density at the face of the slab $n_{\mathrm{H}}$.

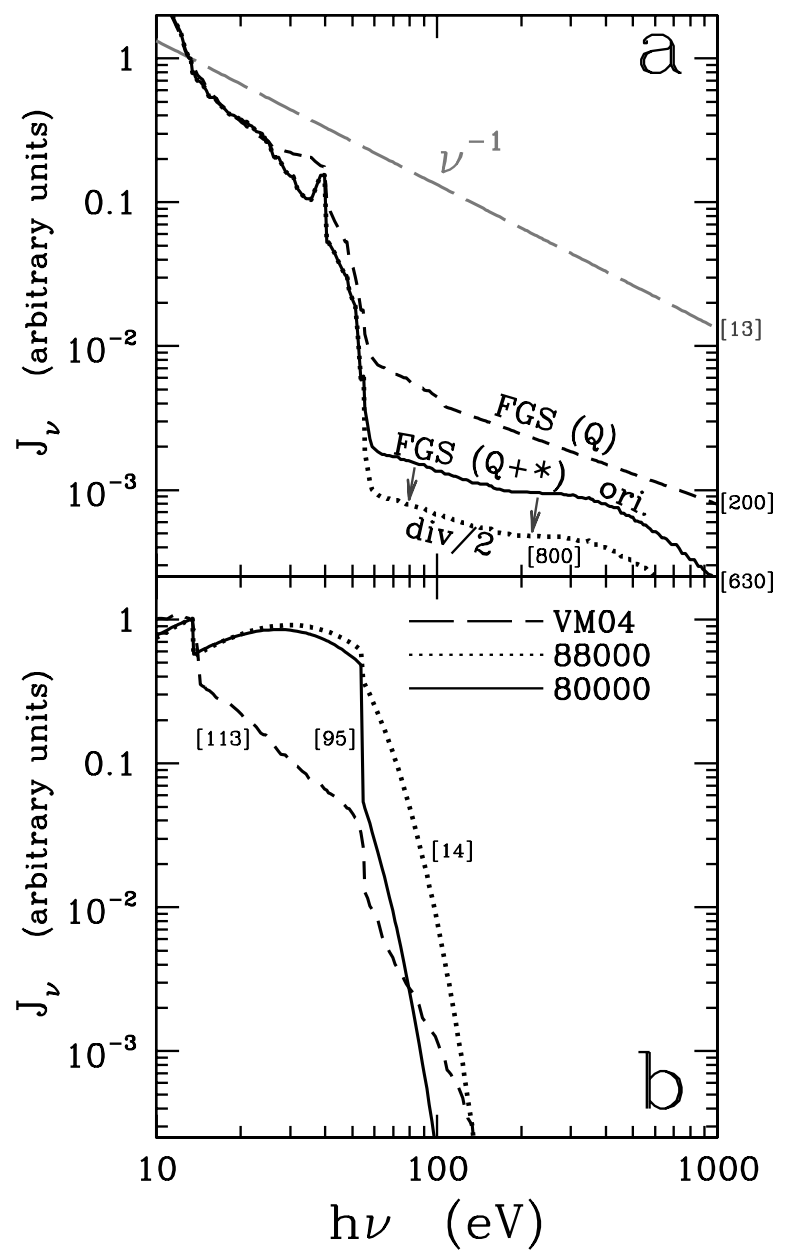

Fig. 1. The spectral energy distribution of various ionizing sources (see Sect. 3) as a function of photon energy. Panel a): silver long-dashed line: spectral energy distribution corresponding to an AGN power-law; short dashed-line: diffuse MBR energy distribution from FGS at $z_{\mathrm{a}}=2$ comprising only quasars $(\mathrm{Q})$ as sources; continuous line: (original) MBR energy distribution from FGS at $z_{\mathrm{a}}=3$ comprising both quasar and stellar sources $(\mathrm{Q}+\star)$; dotted line: same as solid line except that the flux beyond $54.4 \mathrm{eV}$ has been reduced by a factor two. Panel b): continuous line: SED of a zero-age metal-free star of effective temperature $80000 \mathrm{~K}$; dotted-line: SED of a zero-age metal-free star of effective temperature $88000 \mathrm{~K}$; long-dashed line: SED from an evolutionary model of CMK04 corresponding to an age of $3.4 \mathrm{Myr}$ and metallicity of $20 \%$ solar. Values of $\eta$ are given between brackets for each SED (Sect. 3.1).

\subsection{AGN power-law SED}

In the case of direct photoionization by an AGN, we assumed a simple power law of index $\alpha=-1.0$ as in B00 (with $J_{v} \propto v^{+\alpha}$ ) (Fig. 1a).

\subsection{SED of the diffuse metagalactic radiation (MBR)}

The integrated ultraviolet flux arising from distributed QSOs and/or from hot massive stars (in metal-producing young galaxies) is believed to be responsible for maintaining the intergalactic diffuse gas, the Ly $\alpha$ forest, and Lyman limit systems in a highly ionized state. The spectrum and intensity of the diffuse MBR is affected not only by photoelectric absorption from intergalactic matter, but also by the re-emission from radiative recombinations within the absorbing gas itself. In short, QSO 
absorption-line systems are sources, not just sinks of ionizing photons, as shown by Haardt \& Madau (1996).

Detailed calculations of the propagation of QSO and stellar ionizing radiation through the intergalactic space have been presented by Fardal et al. (1998; FGS in the figures or footnotes) and the resulting SEDs relevant to the current work ${ }^{7}$ are shown in Fig. 1a. On the one hand, we have the metagalactic SED in which only quasars are contributing corresponding to model Q2 in their Fig. 7 and, on the other, the SED in which hot stars from star forming regions are included, a model shown in their Fig. 6. In this model, the stars are contributing twice the flux of quasars at $13.6 \mathrm{eV}$. The dotted line is a similar SED, except that the flux beyond 4 Ry has been divided by two. It is an ad hoc model representing the case in which stars are contributing proportionally more with respect to quasars (a similar SED was also considered by Telfer et al. 2002).

The sharp drop in flux at $54.4 \mathrm{eV}$ is a characteristic of all metagalactic radiation models and is due to the cumulative opacity of He II within the IGM. As the IGM SEDs extend into the soft $\mathrm{X}$-rays, it is important to include the harder radiation beyond $\gtrsim 200 \mathrm{eV}$, otherwise the calculated C IV columns are affected, especially when $U$ is large.

If we turn to the values of the softness parameter (Sect. 3.1) observed among IGM absorbers, there is a substantial dispersion in the values measured by Kriss et al. (2001), with $1 \lesssim \eta \lesssim 1000$, which suggests that for a fraction of absorbers, stellar ionizing sources might be contributing. The possibility of a rather inhomogeneous distribution of the SED hardness according to location is favoured by the independent study of Smette et al. (2002), who find that $20 \lesssim \eta \lesssim 5000$.

\subsection{Stellar-ionizing SEDS}

In the stellar ionizing case (panelb in Fig. 1), we considered metal-free stellar SEDs that approximate those studied by Schaerer (2002) with $T_{\text {eff }}$ among one of the following values: $42000,57000,71000,80000$, and $88000 \mathrm{~K}$. In Fig. 1b, we illustrate the cases of the 80000 and $88000 \mathrm{~K}$ SEDs. As in BG03, who presented various photoionization models for the LAN, we approximate the selected stellar SEDs, using a technique that reproduces the ionizing photon luminosities $Q(\mathrm{H}), Q\left(\mathrm{He}^{0}\right)$ and $Q\left(\mathrm{He}^{+}\right)$of the selected $\log T_{\text {eff }}$ model listed in Table 3 of Schaerer (2002). In a similar fashion to Shields \& Searle (1978), we derive the monochromatic temperatures at the edge boundaries $T_{\mathrm{H}^{0}}^{+}, T_{\mathrm{He}^{+}}^{-}$, and $T_{\mathrm{He}^{+}}^{+}$, and then interpolate linearly in $\log T_{v}$ for all the wavelengths used in the code MAPPINGS Ic. We equated $T_{\mathrm{H}^{0}}^{-}$to $T_{\text {eff }}$ and neglected the very small $\mathrm{He}^{0}$ edge present in these atmospheres. This simplified representation of a stellar atmosphere provides enough accuracy to compute the essential properties of the emission line spectrum.

We additionally considered an SED derived from the stellar evolutionary model of Cerviño et al. (2004, hereafter CMK04), which was used by Villar-Martín et al. (2004; VM04) in their photoionization calculations of the LAN. The selected SED corresponds to a metallicity $Z_{*}=0.20 Z_{\odot}$ and an age of $3.4 \mathrm{Myr}$ (Fig. 1b). We included the weak X-ray flux that results from the conversion of the kinetic energy of the supernova remnants into X-ray emission (it did not have any effect on the results). The stellar cluster at that particular age harbours an important population of WR stars and, as shown by VM04, the resulting

\footnotetext{
7 The SEDs calculated by FGS are softer than those of Haardt \& Madau (1996). In their Appendix A, FGS justifies this difference by the more detailed treatment of the cloud opacity and re-emission.
}

ionizing continuum is sufficiently hard to reproduce the emission line strength of the He II $\lambda 1640$ line observed in the LAN spectrum. The CMK04 evolutionary models are characterised by a power-law initial mass function with a Salpeter IMF and stellar masses comprised in the range $2-120 M_{\odot}$.

\section{Model results}

In this section, we present a grid of photoionization calculations for comparison with the observed $N_{\mathrm{CIV}} / N_{\mathrm{HI}}$ ratios in the two HzRGs and the LAN. In Sect. 4.1, we outline our investigative procedure and the format we adopt to display the results. Thereafter, we explore the effects of using different SEDs and varying some of the input parameters, as follows: (a) the power-law photoionization is first studied in Sect. 4.2 assuming different metallicities; (b) in Sect. 4.3 we study various MBR energy distributions in which quasars and stars contribute in different proportions; (c) in Sect. 4.4 we explore stellar photoionization by metal-free atmospheres of varying $T_{\text {eff }}$ and by a stellar cluster SED containing WR stars.

\subsection{Aims and modelling procedure}

There is a gap of more than four orders of magnitude in the $N_{\text {CIV }} / N_{\text {HI }}$ ratio between $0943-242$ and $0200+015$. Rather than explain this with a factor $\sim 1000$ difference in absorber metallicity between the two HzRGs as in J03, we instead explore alternative SEDs. As stated in Sect. 1.5, our practical goal is to find an SED that reduces the metallicity gap to $\sim 10$ (that is, obtaining a successful model that use abundances as low as $\sim 10 \%$ solar). We do not aim at obtaining exact fits of this ratio in each case, but rather at establishing an order of magnitude agreement between the models and the separate observations of the thin and thick absorber categories. For this reason, we only consider the following four widely-spaced metallicities for the haloes: 10 , $1,0.1$ and $0.01 Z_{\odot}$. The higher the metallicity, the higher the $N_{\mathrm{CIV}} / N_{\mathrm{HI}}$ ratio. The proportionality is linear except in the highmetallicity regime, where the slab temperature structure is somewhat altered, this effect being more important in the case of the thick absorbers. No attempt is made in this paper to model the background EELR spectrum. The metallicity of the EELR gas is much higher than (and unrelated to) the absorber's, as discussed by B00.

The ionization parameter characterising the models is plotted on the abscissa in all figures. It is a free parameter that cannot be adequately constrained with the limited data at hand. The target $N_{\mathrm{CIV}} / N_{\mathrm{HI}}$ ratio ( $y$-axis) for each observational datum is represented by a horizontal line, since $U$ is not known. Our aim will be to find models that either cross this observational line or come close to it. We consider it unlikely that $U$ is smaller than 0.001 , since $\mathrm{C}^{+3}$ would then be reduced to a trace species. It is plausible that it takes on much higher values instead, especially in the case of $0200+015$ or the LAN, since high values of $U$ usually result in higher $N_{\mathrm{CIV}} / N_{\mathrm{HI}}$ ratios, a characteristic of these thinner absorbers. We adopt the conservative view that most of the difference between the thin and thick absorbers may be accounted for by differences in the gas excitation, that is, in $U$ rather than by metallicity differences alone. Future observations of other resonance lines might be used to test this (see Sect. 6.1).

In what follows, the slab models presented are either characterized by a small $\mathrm{H}$ I column of $10^{14.8} \mathrm{~cm}^{-2}$, as in $0200+015$ and the LAN, or by a larger column of $10^{19} \mathrm{~cm}^{-2}$ as in $0943-242$. We emphasise that in all the Figs. 2-6 of Sect. 4, the black-line models only apply to the thin absorbers shown at the top while 


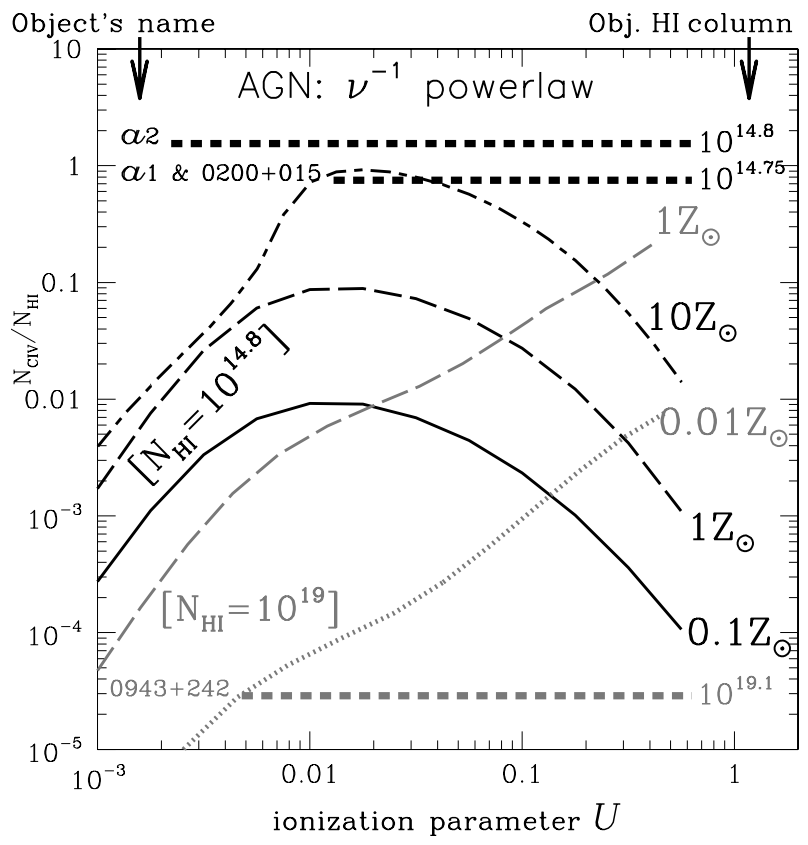

Fig. 2. The column ratio $N_{\mathrm{CIV}} / N_{\mathrm{HI}}$ derived from photoionization by a power law of index -1.0 , as a function of the ionization parameter $U$. Each model sequence (thin lines) along which $U$ varies represents a slab of fixed $N_{\mathrm{HI}}$ column (shown between brackets). Thin black lines connect models of constant column $N_{\mathrm{HI}}=10^{14.8} \mathrm{~cm}^{-2}$, while thin gray lines connect models of constant column $N_{\mathrm{HI}}=10^{19} \mathrm{~cm}^{-2}$. The gas metallicity is shown using labels, in units of $Z_{\odot}$. The thick horizontal broken lines represent four measurements of $N_{\mathrm{CIV}} / N_{\mathrm{HI}}$ : the extended absorbers in the high- $z$ radio galaxies 0943-242 (in gray) and 0200+015 (in black) and the two absorbers found in the lensed Lynx arc nebula [LAN] (in black) and labelled $a 1$ and $a 2$ by Fosbury et al. (2003). The ratios for $0200+015$ and $a 1$ are very similar and have been combined into a single entry. The object's name and the $N_{\mathrm{HI}}$ column appear to the left and right, respectively, of the corresponding horizontal broken line.

the gray-line models only apply to 0943-242 (shown at the bottom).

\subsection{Power-law photoionization}

We present photoionization calculations in Fig. 2 for the case of an AGN power law of index -1.0. Using a moderately different index would not significantly alter the conclusions reached below. For instance, a steeper index -1.4 would only increase the column ratio by a factor of $\lesssim 2$.

In the case of the 0943-242 absorber, the models in Fig. 2 favour abundances much lower than solar, that is of order $1 \%$ solar. B00 favoured a metallicity value of $\simeq 0.02 Z_{\odot}$. A much lower (higher) ionization parameter is a possibility that cannot be ruled out, and the metallicity would then be higher (lower) than the values we considered.

In the case of the $0200+015$ absorber, very high metallicities are favoured by the power-law SED, as found by J03. This is shown in Fig. 2, which suggests a gas metallicity of about ten times solar. We expressed concerns about such high values in Sect. 1.5.

We reject the power-law SED on account of the geometrical considerations presented in Sect. 1.2 that led us to rule out direct ionization by the nuclear radiation from the AGN. The main purpose in reporting power-law calculations is to provide a convenient comparison with the softer continuum shapes explored

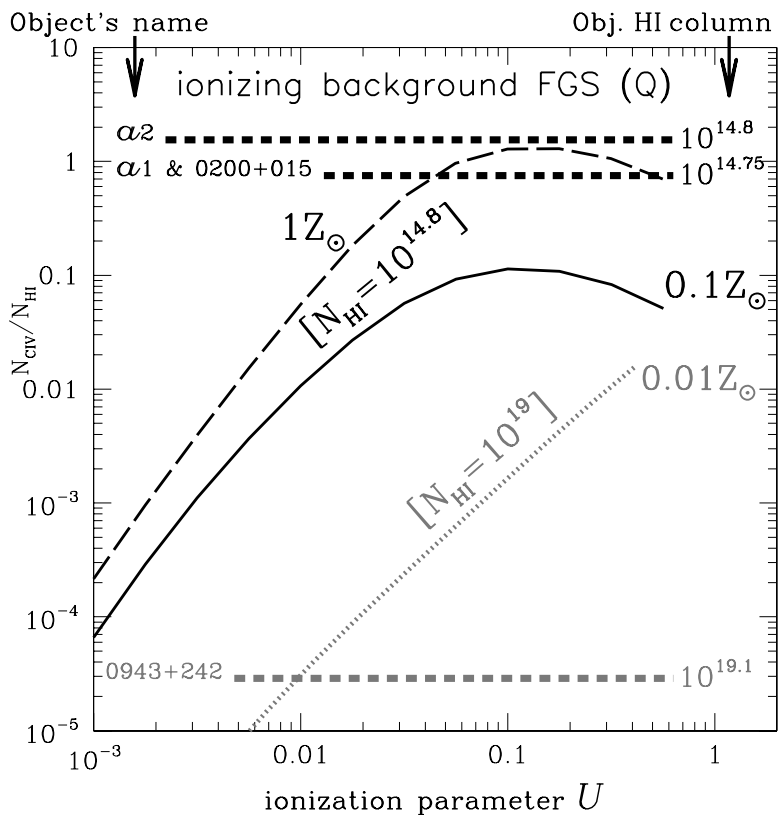

Fig. 3. The column ratio $N_{\mathrm{CIV}} / N_{\mathrm{HI}}$ derived from photoionization by the diffuse MBR due to quasars alone, as a function of $U$. The continuous black line and the long-dashed black line correspond to the thin absorber case assuming metallicities of 0.1 solar and solar, respectively, while the dotted gray line corresponds to the thick absorber case assuming metallicities of 0.01 solar. The nomenclature and symbols have the same meaning as in Fig. 2. In all figures, black line models only apply to the thin absorber objects shown at the top, while gray-line models only apply to 0943-242 below.

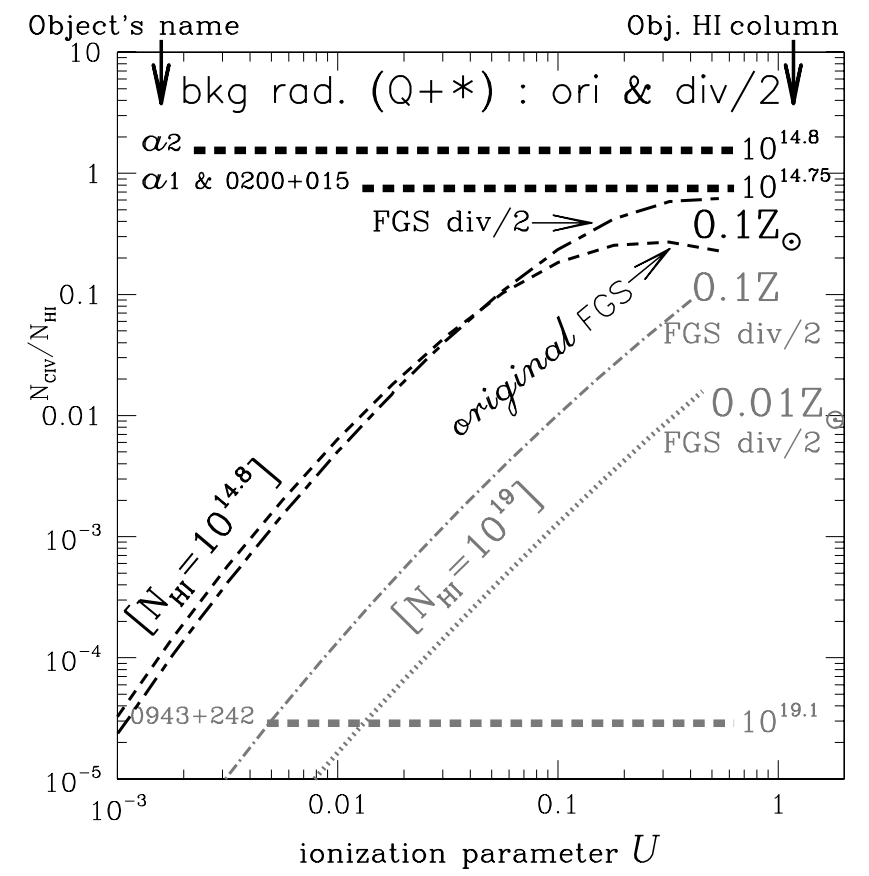

Fig. 4. The column ratio $N_{\mathrm{CIV}} / N_{\mathrm{HI}}$ derived from photoionization by the MBR due to stars and quasars, as a function of $U$. The black short-dashed line corresponds to photoionization by the original FGS SED in which the MBR flux is generated by quasars, as well as stars, assuming a gas metallicity of $0.10 Z_{\odot}$. The black short-long dashed line has an SED similar to the previous one, but its flux beyond $54 \mathrm{eV}$ has been halved (the dotted-line SED in Fig. 1a), owing to a larger contribution by stars. The gray dot-dashed line and gray dotted line assume this latter SED in the thick slab case, but with metallicities of 0.1 and $0.01 Z_{\odot}$, respectively. The nomenclature and symbols have the same meaning as in Fig. 2. 


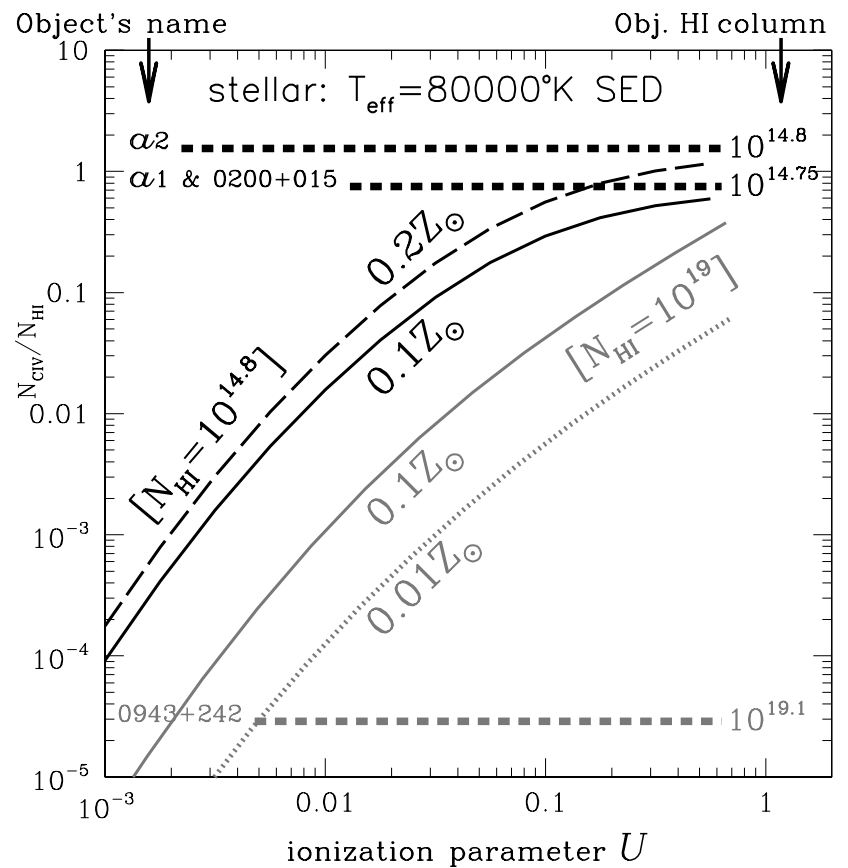

Fig. 5. The column ratio $N_{\mathrm{CIV}} / N_{\mathrm{HI}}$ derived from photoionization by a metal-free stellar atmosphere with $T_{\text {eff }}=80000 \mathrm{~K}$ as described in Sect. 3.4, as a function of $U$. The nomenclature and symbols have the same meaning as in Fig. 2.

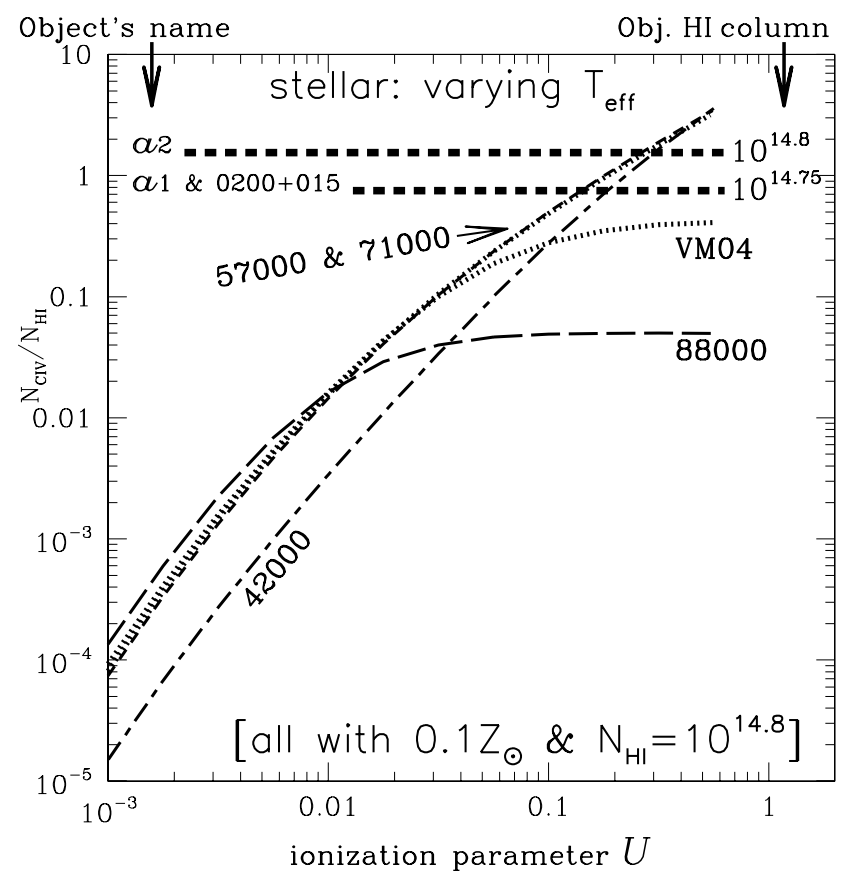

Fig. 6. The column ratio $N_{\mathrm{CIV}} / N_{\mathrm{HI}}$ derived from photoionization by stellar energy distributions of varying $T_{\text {eff }}$, as a function of $U$. In all models, the metallicity is $0.1 Z_{\odot}$ and the slab opacity $10^{14.8} \mathrm{~cm}^{-2}$. A label shows the $T_{\text {eff }}$ under consideration. The dotted line labelled VM04 corresponds to photoionization by a stellar cluster of metallicity $20 \%$ solar and 3.4 Myr of age, as described in Sect.3.4. The nomenclature and symbols have the same meaning as in Fig. 2.

below. We note how different the behaviour of $N_{\mathrm{CIV}} / N_{\mathrm{HI}}$ is between the thin and thick absorber case, in Fig. 2 (compare the two models of solar metallicity).

\subsection{Photoionization by the diffuse MBR}

\subsubsection{MBR flux from quasars alone}

The SED of the diffuse MBR resulting from quasars alone (Fig. 1a) is significantly softer than a power law. Calculations with such an SED are shown in Fig. 3 . The calculated $N_{\mathrm{CIV}} / N_{\mathrm{HI}}$ ratio assuming $10 \%$ solar gas lies below the observed value in $0200+015$, by a factor $\sim 10$, as shown in Fig. 3 . Metallicities about solar would be required so that the model overlaps the observed column ratio ${ }^{8}$. As for 0943-242, in the absence of definite information about $U$, the absorber's metallicity cannot be constrained any further than in the previously covered powerlaw case in Sect. 4.2.

\subsubsection{MBR flux from stars and quasars}

In the case where stars and not just quasars contribute to the MBR, the ionizing SED becomes softer (continuous line in Fig. 1a) and the $N_{\mathrm{CIV}} / N_{\mathrm{HI}}$ ratio observed in $0200+015$ can now be reproduced using metallicities not much above $10 \%$ solar, as illustrated in Fig. 4, as is also the case of an even softer SED in which the flux beyond $54 \mathrm{eV}$ has been halved (see the dotted line SED in Fig. 1a). This latter SED hence satisfies our initial goal defined in Sect. 4.1 with respect to metallicity. But adopting an SED in which star-forming galaxies are contributing more than quasars does not imply that such a distribution is typical of the average MBR. It only suggests that this SED is valid in the neighbourhood of 0200+015. Smette et al. (2002) found, for instance, that the softness of the MBR energy distribution (parameter $\eta$, see Sect. 3.1) presents important local variations, with some locations where only quasars are apparently contributing, while in others there appears to be a significant contribution from starbursting galaxies.

In the case of the thicker absorber in 0943-242, there is little difference in $N_{\mathrm{CIV}} / N_{\mathrm{HI}}$ between the Q+ $\star$ SED in which the flux beyond $54 \mathrm{eV}$ has been halved and the SED produced by quasars only (compare models with $0.01 Z_{\odot}$ in Figs. 3 and 4 ).

In summary, a diffuse MBR sustained by quasars and starforming galaxies is quite successful in reproducing the observed column ratio in $0200+015$ without need for a metallicity any higher than $\sim 0.10 Z_{\odot}$.

\subsection{Photoionization by local stellar UV}

\subsubsection{Photoionization by hot stars with $T_{\text {eff }}=80000 \mathrm{~K}$}

Using a metal-free stellar atmosphere of $80000 \mathrm{~K}$ and a gas metallicity of $4 \%$ solar, BG03 obtained a reasonable first order fit to the strong lines observed in the unusual spectrum of the high redshift LAN. In Fig. 5, we show that the column-density ratios of the LAN absorbers can be reproduced using a high value of $U$ and an absorption gas metallicity of $0.1-0.2 Z_{\odot}$. This range is consistent with the comprehensive metallicity determination of the nebular emission gas by VM04, that is $\simeq 10 \%$ solar. Given the similarity of the LAN column ratio with that of $0200+015$, we infer that a $N_{\mathrm{CIV}} / N_{\mathrm{HI}}$ ratio of order unity in an $\mathrm{Hz}$ RG is compatible with a stellar SED photoionizing a subsolar metallicity absorber. Hence, the possibility that the $0200+015$ absorber might be photoionized by hot stars warrants consideration, since metallicities of only $10 \%$ solar would be needed rather than a value

\footnotetext{
${ }^{8}$ Hence in the thin slab case, the increase in $N_{\mathrm{CIV}} / N_{\mathrm{HI}}$ provided by a quasar-based MBR SED is a factor ten with respect to a straight AGN power law.
} 
100 times higher as favoured by the power-law SED (Fig. 2 or J03). The problem of stellar continuum detection is discussed in Sect. 5.2.2.

In the case of thicker absorbers, as represented by 0943-242, the gray-line models point to metallicities in the range $0.01-0.1 Z_{\odot}$, assuming $U<0.05$. Higher values of $U$ would imply lower absorber metallicities. Interestingly, the stellar (Fig. 5) and the power-law (Fig. 2) models with $0.01 Z_{\odot}$ cross the $N_{\mathrm{CIV}} / N_{\mathrm{HI}}$ ratio of $0943-242$ at a very similar $U$ value. This indicates that thicker slabs are much less sensitive to the SED's shape.

\subsubsection{Varying the stellar atmosphere temperature}

In Fig. 6 we explore the effect of varying stellar effective temperature. The zero-age metal-free atmospheres used correspond to $T_{\text {eff }}$ of $42000,57000,71000,80000$, and $88000 \mathrm{~K}$ (from Schaerer 2002). All models are characterised by a column $N_{\mathrm{HI}}=$ $10^{14.8} \mathrm{~cm}^{-2}$ and a gas metallicity of $10 \%$ solar appropriate to the LAN. Although temperatures lower than $80000 \mathrm{~K}$ can easily fit the LAN column ratio, this would imply too weak an He II $\lambda 4686$ emission for the nebula. At the other temperature end, a $T_{\text {eff }}$ as high as $88000 \mathrm{~K}$ would require a ten times higher gas metallicity in order to reproduce the observed column ratio. The reason is that, as $T_{\text {eff }}$ is increased much beyond $70000 \mathrm{~K}$, the increase in the continuum's hardness causes the slab to harbour many ionization stages of carbon (e.g. $\mathrm{C}^{+4}$ and $\mathrm{C}^{+5}$ ), thereby causing a relative reduction of the $\mathrm{C}$ IV fraction. Increasing the temperature much beyond $10^{5} \mathrm{~K}$ would result in column ratios approaching those of a power-law.

VM04 have modelled the LAN emission line spectrum using the ionizing spectrum of an evolved stellar cluster in which transient Wolf-Rayet stars can account for the nebular He II $\lambda 4686$ line observed in emission. A photoionization model of the absorber using such an SED is represented by the dotted line labelled VM04 in Fig. 6. The behaviour of the column ratio is similar to that of a $80000 \mathrm{~K}$ metal-free star (Fig. 5).

In the case of the HzRG shells, stellar SEDs are possible candidates for the ionization of the absorbers (but not of their EELR), since they can reproduce the observed column ratio using subsolar metallicities.

\section{Estimates of the radii and masses of the shells and compatibility with other observables}

Armed with the results of the photoionization calculations, we now investigate two of the scenarios in more detail, namely the cases of ionization by the MBR and by hot stars. We focus on their implications for other properties of the absorbing shells (e.g. mass, radius, and thickness) and their compatibility with other observables (e.g. the underlying stellar continuum in the case of ionization by hot stars and the strength of the Ly $\alpha$ emission). On the basis of such considerations we demonstrate that ionization by hot stars is favoured over MBR ionization. Readers who do not wish to follow the argument in full may skip over Sects. 5.1 and 5.2 and proceed directly to the summary in Sect. 5.3.

\subsection{The case of MBR ionization}

We first analyse the possibility of having the MBR ionize the haloes. The diffuse MBR is ubiquitous and its intensity independent of distance to the $\mathrm{HzRG}$; therefore, changes in excitation (i.e. $U$ ) are obtained by varying the gas density. Higher shell densities, hence lower $U$, might explain the absence of C IV absorption in many $\mathbf{H} z \mathbf{R G}$ shells. We must thus investigate whether the MBR is strong enough to result in an acceptable halo density, because the geometrical thickness of the shell increases as the density is reduced. Below we use such constraints to infer the shell's minimum distance from the $\mathrm{HzRG}$ and its total mass.

\subsubsection{MBR intensity and shell thickness}

An estimate of the MBR mean intensity is provided by the proximity effect, whereby absorbers becoming more ionized in the vicinity of quasars. We adopt the value $J_{v} \approx$ $10^{-21} \mathrm{erg} \mathrm{cm}^{-2} \mathrm{~s}^{-1} \mathrm{~Hz}^{-1} \mathrm{sr}^{-1}$ inferred by Cooke et al. (1997) and assume the MBR flux SED Q+ + of Fardal et al. (1998), albeit with the flux above 4 Ry divided by two, as studied in Sect. 4.3.2.

We consider the two cases of the weak and the strong absorber cases, using 0200+015 and 0943-242 as examples, respectively. Using the definition ${ }^{9}$ of $U$ and MAPPINGS Ic to integrate the $\mathrm{Q}+\star \mathrm{SED}$, we find in the optically thin case that the total hydrogen density is given by $n_{\mathrm{H}}^{\text {thin }}=2.8 \times$ $10^{-4} J_{-21}\left\{U_{0.1}^{\text {thin }}\right\}^{-1} \mathrm{~cm}^{-3}$, where $U_{0.1}^{\text {thin }}=U^{\text {thin }} / 0.1$ and $J_{-21}=$ $J_{v} /\left(10^{-21} \mathrm{erg} \mathrm{cm}^{-2} \mathrm{~s}^{-1} \mathrm{~Hz}^{-1} \mathrm{sr}^{-1}\right)$. In the case of thicker absorbers with $N_{\mathrm{H}} \gtrsim 10^{18} \mathrm{~cm}^{-2}$, because of self-shielding, illumination of a spherical shell can only occur from the outside, and the mean intensity is approximately half of the previous thin case, such that $n_{\mathrm{H}}^{\text {thick }}=2.8 \times 10^{-3} J_{-21}\left\{U_{0.005}^{\text {thick }}\right\}^{-1} \mathrm{~cm}^{-3}$, where (for convenience) $U_{0.005}^{\text {thick }}=U^{\text {thick }} / 0.005$. The photoionization calculations at constant $N_{\mathrm{HI}}$ (either $10^{14.8}$ or $10^{19} \mathrm{~cm}^{-2}$ ) indicate that the total hydrogen column of the slab can be approximated as $N_{\mathrm{H}}^{\text {thin }} \simeq 2.1 \times 10^{19}\left\{U_{0.1}^{\text {thin }}\right\}^{1.1} \mathrm{~cm}^{-2}$ and $N_{\mathrm{H}}^{\text {thick }} \simeq$ $6.3 \times 10^{20}\left\{U_{0.005}^{\text {thick }}\right\}^{1.1} \mathrm{~cm}^{-2}$, respectively. As argued in Sect. 1.3, a shell geometry is more appropriate than that of a filled sphere. We therefore introduce an aspect ratio $A=\Delta r / r$ for the shell, where $\Delta r$ is the shell thickness and $r$ its outer radius, taking the HzRG nucleus as the centre. Since this ratio is not known, we define an upper limit of $A \lessgtr 0.2$. Since the total column density is given by $N_{\mathrm{H}}=r n_{\mathrm{H}} A$, this limit on $A$ translates into a lower limit for the shell radius (i.e. a minimum radius) of $r_{\mathrm{kpc}}^{\text {thin }} \geq 122\left\{U_{0.1}^{\text {thin }}\right\}^{2.1}\left\{A_{0.2} J_{-21}\right\}^{-1} \mathrm{kpc}$ and $r_{\mathrm{kpc}}^{\text {thick }} \geq$ $365\left\{U_{0.005}^{\text {thick }}\right\}^{2.1}\left\{A_{0.2} J_{-21}\right\}^{-1} \mathrm{kpc}$, respectively, with $A_{0.2}=A / 0.2$. To be definite, we will assume that both absorbers have the same gas metallicity of $0.10 Z_{\odot}$. From Fig. 4 , we read off values of $U_{0.1}^{\text {thin }} \simeq 2$ and $U_{0.005}^{\text {thick }} \simeq 1$ for $0200+015$ and $0943-242$, respectively. This translates into minimum radii of 523 and $365 \mathrm{kpc}$, respectively. Hence MBR ionization implies that the shells are extremely distant from the background EELR, and this appears to be difficult to reconcile with the observations that show a distinct transition from sources with radio extents $<25 \mathrm{kpc}$ to those with large radiosizes (see Sect. 5.2.1).

Since $r_{\mathrm{kpc}} \propto U^{2.1}$, smaller radii follow from assuming lower values of $U$, which would require that we adopt metallicities somewhat higher than $0.1 Z_{\odot}$ (higher metallicities shift models to the left in Fig. 4). Uncertainties in $U$ (or equivalently in $Z$ ) therefore affect our estimates of the shell's geometrical thickness significantly. In Sects. 6.1 and 6.2, we indicate how detection of the shells in $\mathrm{Mg}$ II or O VI would help to constrain both $Z$ and $U$.

\footnotetext{
9 If the SED were a power law of index $\alpha(<0)$, we would have $U \approx$ $-4 \pi J_{v} /\left(n_{\mathrm{H}} h c \alpha\right)=6.310^{-5} J_{-21} / n_{\mathrm{H}}$, where $h$ is the Planck constant and $c$ the speed of light.
} 


\subsubsection{Problem of the high shell masses}

Since the shell masses are given by $M_{\mathrm{H}}=4 \pi r^{2} m_{\mathrm{H}} N_{\mathrm{H}}=$ $10^{-13} r_{\mathrm{kpc}}^{2} N_{\mathrm{H}} M_{\odot}$, assuming they are spherical, we can use the previous expressions for the minimum radii to derive the following minimum masses $M_{\mathrm{H}}^{\text {thin }} \geq 3.1 \times 10^{10}\left\{U_{0.1}^{\text {thin }}\right\}^{5.3}\left\{A_{0.2} J_{-21}\right\}^{-2} M_{\odot}$ and $M_{\mathrm{H}}^{\text {thick }} \geq 8.3 \times 10^{12}\left\{U_{0.005}^{\text {thick }}\right\}^{5.3}\left\{A_{0.2} J_{-21}\right\}^{-2} M_{\odot}$, respectively. Adopting the same estimates of $U$ as above, we derive masses of $M_{\mathrm{H}}^{\text {thin }} \geq 1.2 \times 10^{12} M_{\odot}$ and $M_{\mathrm{H}}^{\text {thick }} \geq 8.3 \times 10^{12} M_{\odot}$ for $0200+015$ and 0943-242, respectively. At face value, these values are excessive and would suggest that MBR ionization is unworkable. On the other hand, given the strong dependence of $M_{\mathrm{H}}$ above on poorly determined quantities, the upper limits mentioned above are order-of-magnitude estimates and, as such, do not allow us to completely rule out MBR ionization. For instance, reducing both ionization parameters by two reduces the $0200+015$ and 0943-242 shell mass estimates to $3.1 \times 10^{10}$ and $2.1 \times 10^{11} M_{\odot}$, respectively. Proportionally lower masses would be implied if the shells covered only a fraction of $4 \pi \mathrm{sr}$. On the other hand, if the shells were geometrically very thin (i.e. $A_{0.2} \ll 0.2$ ), the mass of the 0943-242 shell would become unreasonably high ( $\left.>10^{13} M_{\odot}\right)$.

In conclusion, the ionization of the shells by the diffuse MBR would imply that the shells have expanded to large distances from the parent HzRG. This would favour the "aging shell" scenario B. A significant problem is that the shell-mass estimates turn out too large. An alternative is that the ionizing radiation is stronger as a result of local stellar sources, as discussed below.

\subsection{The case of ionization by local stellar sources}

The similarity of the $N_{\mathrm{CIV}} / N_{\mathrm{HI}}$ ratio between the stellar-excited LAN nebula and the $0200+015$ absorber suggests that hot stars could be the ionization source of the HzRGs haloes. Even though the emission-line spectra of the background EELR is clearly AGN-like and presumably ionized by the hidden quasar, an interesting result of the calculations in Sect. 4.4 is that the column ratios in the two $\mathrm{HzRG}$ absorbers can be reproduced using a stellar SED and subsolar metallicites, as for the LAN. We now analyse some of the implications of this hypothesis.

\subsubsection{Test case: hot stars contributing little to the EELR}

The geometry that we envisage is that of a large population of hot stars, possibly distributed uniformly or in large aggregates as a result of merging (e.g. the $\mathrm{HzRG} 4 \mathrm{C} 41.17$; van Breugel et al. 1997). To simplify the treatment of the geometrical dilution of the ionizing radiation, we assume that the propagation of the photons is approximately radial by the time they reach the intervening shells. To facilitate the comparison with the previous MBR ionization case, we define a reference test case with a much higher shell density of $0.01 \mathrm{~cm}^{-3}$. The photon density is set by the relation $n_{\mathrm{H}}=10^{-2}\left\{U_{0.1}\right\}^{-1} \mathrm{~cm}^{-3}$, which is equivalent to having an ionizing flux (reaching the shell) 36 times higher than provided by the MBR intensity with $J_{-21}=1$, as assumed in Sect. 5.1.1. Under the conditions of this test case, our calculations indicate that the ionizing photon flux impinging upon the inner boundary of the shells is $\varphi_{\mathrm{H}}=3.0 \times 10^{7} n_{0.01} U_{0.1}$ quanta cm ${ }^{-2} \mathrm{~s}^{-1}$, where $n_{0.01}=n_{\mathrm{H}} / 0.01$ represents the shell density. Local stellar sources (in contrast to the MBR case) accord better with the "inner shell" scenario A, in which the shells do not extend farther out than about $25 \mathrm{kpc}$ in radius, i.e. the apparent crossover point between sources with absorbers and those without (see J03; W04; Sect. 1.1 and the superwind-bowshock model of Krause 2005). The photon luminosity is $4 \pi r^{2} \varphi_{\mathrm{H}}$, which can be written as $Q_{\mathrm{H}}=0.224 \times$ $10^{55} r_{25}^{2} n_{0.01} U_{0.1}$ quanta s$^{-1}$, where $r_{25}=r_{\mathrm{kpc}} / 25$. The Ly $\alpha$ luminosity from recombination alone is given by the expression $L_{\mathrm{Ly} \alpha}=1.06 \times 10^{-11} \epsilon_{\mathrm{neb}} Q_{\mathrm{H}} \mathrm{erg} \mathrm{s}^{-1}$, where the conversion factor ${ }^{10}$ assumes case $B$ and a temperature of $20000 \mathrm{~K}$. Here, $\epsilon_{\text {neb }}$ is the fraction of photons absorbed and reprocessed by the emission gas. The leaking fraction $1-\epsilon_{\text {neb }}$ for very luminous H II regions lies in the range 0.3-0.5 (Beckman et al. 2000 and references therein; Zurita et. al. 2002; Relaño et al. 2002; Giamanco et al. 2005). To be definite, we adopt 0.5 and define $\epsilon_{0.5}^{\text {neb }}=\epsilon_{\text {neb }} / 0.5$ to find that

$L_{\mathrm{Ly} \alpha}=0.12 \times 10^{44} \epsilon_{0.5}^{\mathrm{neb}} r_{25}^{2} n_{0.01} U_{0.1} \mathrm{erg} \mathrm{s}^{-1}$

for our test case.

The $L_{\mathrm{Ly} \alpha}$ luminosity in the test case should be compared with the significantly higher EELR $L_{\mathrm{Ly} \alpha}$ luminosities of $1.2 \times 10^{44}$ and $1.9 \times 10^{44} \mathrm{erg} \mathrm{s}^{-1}$, observed in $0200+015$ and $0943-242$, respectively. In the case of the LAN, with $L_{\mathrm{Ly} \alpha}=0.40 \times 10^{44} \mathrm{erg} \mathrm{s}^{-1}$, its luminosity ${ }^{11}$ is three times higher than our test case. As for our two EELRs, they are brighter in Ly $\alpha$ by a factor $5(0200+015)$ and 300 (0943-242), assuming in Eq. (1) that $U_{0.1}^{\text {thin }} \simeq 2$ and $U_{0.005}^{\text {thick }} \simeq 1$, respectively. The assumed stellar ionizing luminosity is therefore not expected to alter the AGN character of the HzRG emission spectrum, even though specific emission lines would be subject to a contribution from the proposed stellar sources.

Interestingly, the Ly $\alpha$ luminosities of the absorption shells themselves are expected to be relatively low. We derive Ly $\alpha$ luminosities of $L_{\mathrm{Ly} \alpha}=5.5 \times 10^{43} \epsilon_{\text {shell }} n_{0.01} U_{0.1} \mathrm{erg} \mathrm{s}^{-1}$, where $\epsilon_{\text {shell }}$ is the fraction of ionizing photons absorbed by the shell, a quantity that is set by the shell opacity. Our calculations with $N_{\mathrm{HI}}$ of $10^{14.8}$ and $10^{19} \mathrm{~cm}^{-2}$ indicate that $\epsilon_{\text {shell }}=5 \times 10^{-4}$ and 0.97 for the thin and thick absorbers, respectively. Assuming as in Sect. 5.1.1 that $U_{0.1}^{\text {thin }} \simeq 2$ and $U_{0.005}^{\text {thick }} \simeq 1$ for $0200+015$ and 0943-242, respectively, this translates into the corresponding luminosities of $5.5 \times 10^{40}$ and $2.7 \times 10^{41} \mathrm{erg} \mathrm{s}^{-1}$. These values are negligible with respect to the observed HzRG and LAN Ly $\alpha$ luminosities.

The total column densities for the masses of the shells as a function of $U$ in the case of stellar SED are as follows: $N_{\mathrm{H}}^{\text {thin }} \simeq$ $1.1 \times 10^{19}\left\{U_{0.1}^{\text {thin }}\right\}^{1.1} \mathrm{~cm}^{-2}$ and $N_{\mathrm{H}}^{\text {thick }} \simeq 7.2 \times 10^{20}\left\{U_{0.005}^{\text {thick }}\right\}^{1.1} \mathrm{~cm}^{-2}$, assuming the $80000 \mathrm{~K}$ SED. We used the expression $M_{\mathrm{H}}=$ $6.25 \times 10^{-11} r_{25}^{2} N_{\mathrm{H}} M_{\odot}$, assuming again that the shells are spherical, to derive mass estimates of $M_{\mathrm{H}}^{\text {thin }}=6.7 \times 10^{8} r_{25}^{2}\left\{U_{0.1}^{\text {thin }}\right\}^{1.1} M_{\odot}$ and $M_{\mathrm{H}}^{\text {thick }}=4.5 \times 10^{10} r_{25}^{2}\left\{U_{0.005}^{\text {thick }}\right\}^{1.1} M_{\odot}$ for $0200+015$ and

${ }^{10}$ Under the quoted physical conditions, $65 \%$ of the recombinations lead to Ly $\alpha$ photon emission (e.g. Binette et al. 1993).

11 The observed $L_{\mathrm{Ly} \alpha}$ values quoted above were derived using the Ly $\alpha$ fluxes reported by VO97 and by Fosbury et al. (2003) for the LAN. We assumed the objects to be isotropic emitters and corrected the fluxes for Ly $\alpha$ absorption due to the absorbing shells. For the LAN, $L_{\mathrm{Ly} \alpha}$ was divided by 10 to compensate for the amplification by the gravitational lens. We adopted the concordance $\Lambda \mathrm{CDM}$ cosmology with parameter values of $\Omega_{\Lambda}=0.7, \Omega_{M}=0.3, h=0.70$, where $h=H_{0} / 100$. The isotropic photon luminosities that we infer for $0200+015,0943-242$, and the LAN are $Q_{\mathrm{H}}=1.1 \times 10^{55}, 1.8 \times 10^{55}$, and $0.37 \times 10^{55}$ quanta s$^{-1}$, respectively. These values are lower limits, since they only represent the fraction absorbed by the gas and reprocessed into line emission. To recover the intrinsic $Q_{\mathrm{H}}$, they would have to be increased by $\epsilon_{\text {neb }}{ }^{-1}$, a poorly determined quantity in AGN $\left(\epsilon_{\text {neb }}^{\mathrm{AGN}} \lesssim 0.1\right.$ : Oke \& Korycansky 1982; Antonucci et al. 1989). 
0943-242, respectively. Hence, the shell masses for the test case are quite low in comparison with the MBR case because of the smaller radii implied by the stronger ionizing flux. By the same token, higher densities are implied, which result in shells that are also geometrically very thin $\left(A_{0.2} \ll 1\right)$. The absorber's density can be quite different than the assumed test case with $n_{0.01}=1$. The required stellar luminosity, however, must then scale in the same proportion. For instance, an absorber with density $0.1 \mathrm{~cm}^{-3}$ would require a 10 times higher stellar luminosity. This would cause the nebular lines to be comparable in luminosity to the observed EELR, which would clearly not be desirable.

\subsubsection{On the detection of stellar continuum}

For the case where hot stars alone ionize the foreground absorbers, we now estimate the implied stellar flux (or, equivalently, the Ly $\alpha$ equivalent-width) and compare it with the observations, beginning with the LAN.

Assuming a Salpeter IMF and the SED for an instantaneous burst of age 3.4 Myr (VM04), we find using MAPPINGS Ic that the rest-frame Ly $\alpha$ equivalent-width is $E W_{\text {Ly } \alpha}^{\text {rest }}=190 \epsilon_{\text {neb }} \AA$. Defining the fraction of ionizing photons reprocessed by the emission nebula as $\epsilon_{0.5}^{\text {neb }}=\epsilon_{\text {neb }} / 0.5$, we find that the (observerframe) continuum flux is $F_{\mathrm{c}}^{\mathrm{obs}}=0.0105\left\{\epsilon_{0.5}^{\mathrm{neb}}\left(1+z_{\mathrm{e}}\right)\right\}^{-1} F_{\mathrm{Ly} \alpha}^{\mathrm{obs}} \AA^{-1}$, where $F_{\mathrm{Ly} \alpha}^{\mathrm{obs}}$ is the observed line flux, corrected for absorption.

For the LAN, this implies a $5300 \AA$ continuum of $F_{\mathrm{c}}^{\mathrm{obs}}=$ $9.4 \times 10^{-18} \mathrm{erg} \mathrm{cm}^{-2} \mathrm{~s}^{-1} \AA^{-1}$, or equivalently, $8.8 \mu \mathrm{Jy}$. (The lens amplification was assumed to be the same for both the continuum and the lines). This flux is about 30 times higher than the upper limit set by Fig. 5 of Fosbury et al. (2003), of $\approx 0.3 \mu \mathrm{Jy}$. A continuum was detected at longer wavelengths, but these authors report that it is consistent with being nebular in nature. As a solution, Fosbury et al. (2003) proposed a top-heavy IMF. Assuming a single $T_{\text {eff }}$ SED of $80000 \mathrm{~K}$ (Fig. 1), we derive $E W_{\mathrm{Ly} \alpha}^{\mathrm{rest}}=1335 \epsilon_{\mathrm{neb}} \AA$ or a continuum of $1.3 \mu \mathrm{Jy}\left(\epsilon_{0.5}^{\mathrm{neb}}=1\right)$. Even for a higher $T_{\text {eff }}$ of $88000 \mathrm{~K}$, we obtain a value of $1.1 \mu \mathrm{Jy}$, similar to before. The increase of a factor 7-8 in the Ly $\alpha$ equivalentwidths provided by these two SEDs is therefore insufficient. A significantly hotter stellar SED is therefore required (Fosbury et al. proposed $\simeq 10^{5} \mathrm{~K}$ ). Alternative explanations might consist of a peculiar dust distribution that selectively absorbs the continuum and thereby increases the observed equivalent-width or of differential amplification of the lines due to the gravitational lens (MV04). It is interesting to note that the Ly $\alpha$-emitting "blobs" associated with Lyman break galaxies likewise do not show the expected level for the stellar continuum (Steidel et al. 2000). For a possible explanation involving significant populations of metal-free stars, see Jimenez \& Haiman (2006).

If we now turn to the two HzRGs, we place upper limits on the underlying stellar continua by defining limits on the contribution of hot stars to the EELR Ly $\alpha$ (which must be much smaller than the AGN contribution). Assuming the VM04 SED and the stellar contribution to be no more than $10 \%$ of the EELR, we derive continuum fluxes (at the observed Ly $\alpha$ wavelength) of $7.0 \times 10^{-19}$ and $1.0 \times 10^{-18} \mathrm{erg} \mathrm{cm}^{-2} \mathrm{~s}^{-1} \AA^{-1}$, for $0943-242$ and $0200+015$, respectively ${ }^{12}$. These lie below the upper

\footnotetext{
${ }^{12}$ It should be emphasised that these are maximum estimates of the stellar continuum. We recall that we can let the stellar continuum be much weaker than the test case explored in Sect. 5.2.1 and still have it ionize the shells. Furthermore, if $U \ll 0.1$, as considered for $0200+015$ in Sect.4.4.1, an even weaker continuum is needed, as shown by the estimates of $\operatorname{Ly} \alpha$ luminosity reported in Sect. 5.2.2.
}

continuum limits of $3.1 \times 10^{-18}$ and $2.6 \times 10^{-18} \mathrm{erg} \mathrm{cm}^{-2} \mathrm{~s}^{-1} \AA^{-1}$, respectively, as measured by van Ojik (1995). However, using recent VLT data from VIMOS-IFU (van Breukelen et al. 2005), one of us (MJ) reports detection of the underlying continuum in 0943-242 at the level of $1.7 \pm 0.9 \times 10^{-18} \mathrm{erg} \mathrm{cm}^{-2} \mathrm{~s}^{-1} \AA^{-1}$, that is, less than a factor two above our limit. Vernet et al. (2001) reports on the measurement of a far-UV continuum in the form of "single peaked sources". This continuum, however, is $6.6 \%$ polarized near $1350 \AA$. Vernet et al. (2001) estimates that the AGN contributes between $27 \%$ and $66 \%$ of the continuum at $1500 \AA$. After allowing for a $20 \%$ contribution from the nebular continuum, these authors conclude that between 14 and $55 \%$ of the unpolarized continuum might be due to young stars. The continuum measured by $\mathrm{MJ}$ is then fortuitously consistent with our upper limit, since half of it or less is stellar in origin. We conclude that an instantaneous burst with a Salpeter IMF is thus a feasible source of ionization for $\mathrm{H} z \mathrm{RG}$ absorbers. It would, in any case, be difficult to rule it out since continua much weaker than assumed in our test case (by a factor $\sim 20$ ) would still suffice to ionize the absorbers.

\subsection{Summary of current constraints on $\mathrm{HzRG}$ halo ionization}

To summarise the results of the previous two sections, we conclude that ionization by the MBR or by hot stars can satisfactorily reproduce the observed $N_{\mathrm{CIV}} / N_{\mathrm{HI}}$ ratios without recourse to excessive galaxy-to-galaxy metallicity variations. That was the aim of the photoionization modelling as defined in Sect. 4.1.

On closer inspection, however, ionization by the MBR leads to excessively large radii for the absorbing shells and, by implication, to very high gas masses. This follows because the intensity of the MBR, $J_{v}$, is not a free parameter so constraints on $U$ translate directly into constraints on halo gas density. Given the latter, the observed requirement for a shell-like geometry translates directly into a minimum shell radius from the parent $\mathrm{H} z \mathrm{RG}$. For both thick and thin absorbers, the minimum radii are of the order of several hundred kpc. This is hard to reconcile with the observed transition in radio-source size between HzRGs with and without strong absorption. After scaling as the square of the radius, the implied shell masses are also uncomfortably high.

The case of ionizing the absorber, but not the EELR, by hot stars circumvents the above problems, but at first sight raises separate issues of its own. The first is to ensure that these hot stars do not overproduce the Ly $\alpha$ emission, because in $\mathrm{Hz}$ RGs the EELR is powered by AGN photoionization or jet interactions. In both $0200+015$ and 0943-242, it was shown that Ly $\alpha$ emission from hot stars does not significantly contaminate the EELR emission. Potentially more serious is the apparent faintness of the stellar continuum, which is hard to explain away with peculiar dust geometries if the stars ionize gas in the direction of the observer. For the LAN Fosbury et al. (2003) appealed to hot stars and a top-heavy IMF; for the two HzRGs, constraints on the continuum level below Ly $\alpha$ appear to be consistent with the levels expected from hot stars. For all these reasons, we thus favour hot stars as the more likely source of ionization for the HzRG haloes and in the next section outline some new diagnostics to test this further.

\section{New diagnostics for future observations}

In order to resolve pending issues such as the size, masses, and nature of the $\mathrm{H} z \mathrm{RG}$ haloes, more extensive observations are needed and measurements of C IV in absorption in other HzRGs 


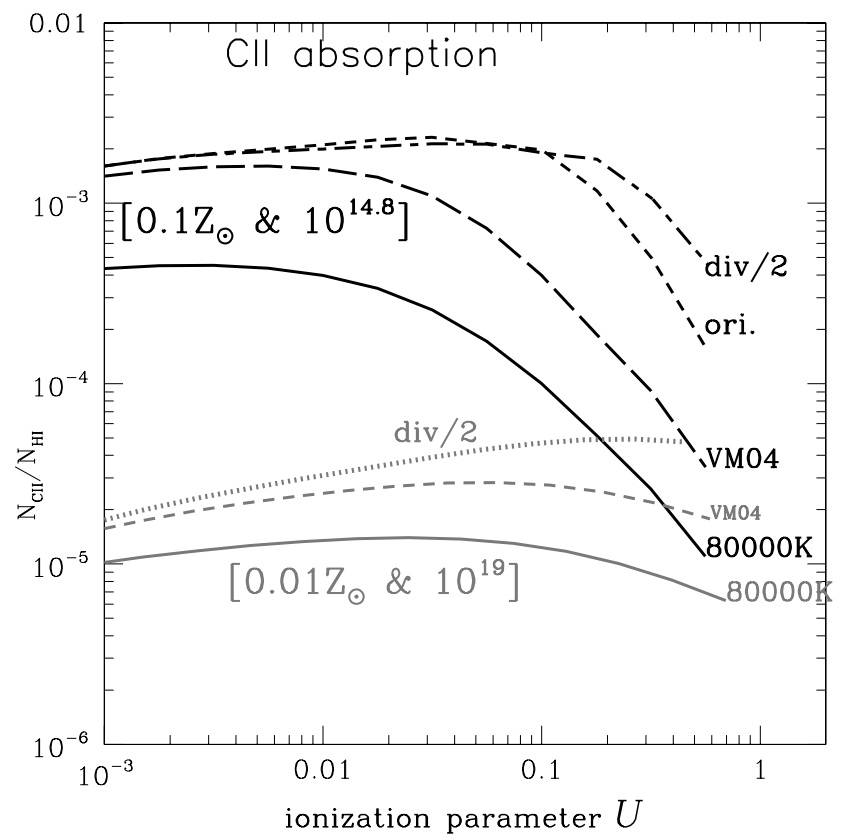

Fig. 7. The column ratio $N_{\mathrm{CII}} / N_{\mathrm{HI}}$ derived from photoionization by four different SEDs discussed in Sect. 4, as a function of $U$. The nomenclature and symbols have the same meaning as in Fig. 2.

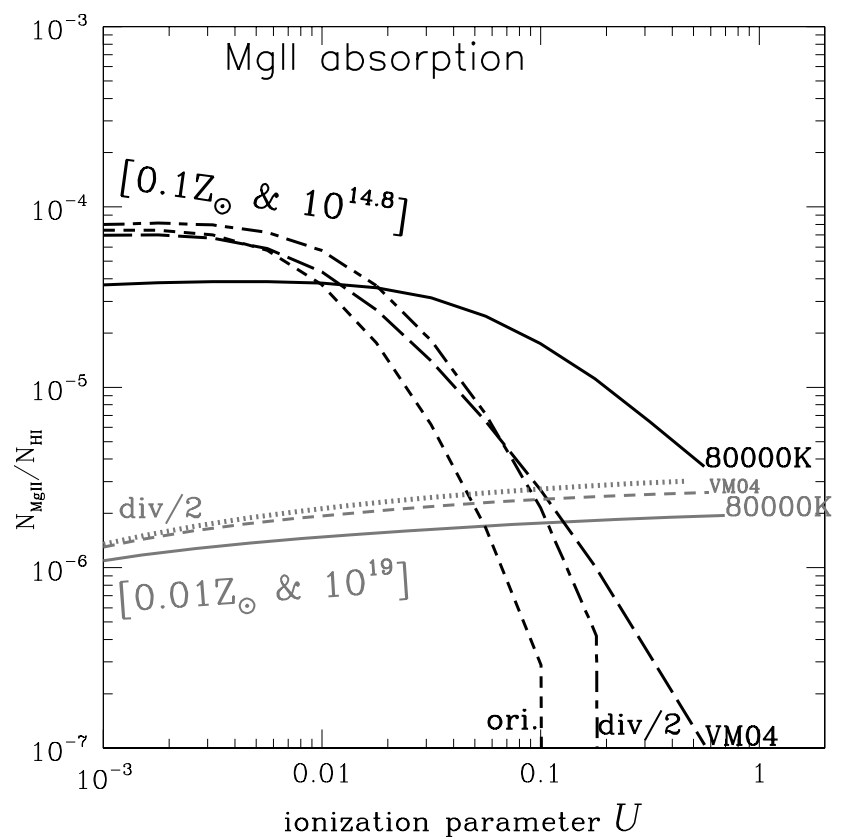

Fig. 8. The column ratio $N_{\mathrm{MgII}} / N_{\mathrm{HI}}$ derived from photoionization by four different SEDs discussed in Sect. 4, as a function of $U$.

should be attempted. The detection of other absorption species would also help to break the $Z-U$ degeneracy, as outlined below.

\subsection{Absorption by lower ionization species: C $11 \lambda \lambda 1335$ and $\mathrm{Mg} \| \lambda \lambda 2798$}

It would be helpful to detect the absorption of other resonance lines in the spectra of HzRGs, particularly in the case of species of lower ionization than CIV. This could be used to confirm whether those $\mathrm{HI}$ absorbers without C IV absorption might simply correspond to shells of lower ionization (smaller $U$ ). Two candidate species are $\mathrm{C}$ II $\lambda \lambda 1335$ and $\mathrm{Mg}$ II $\lambda \lambda 2798$. We report

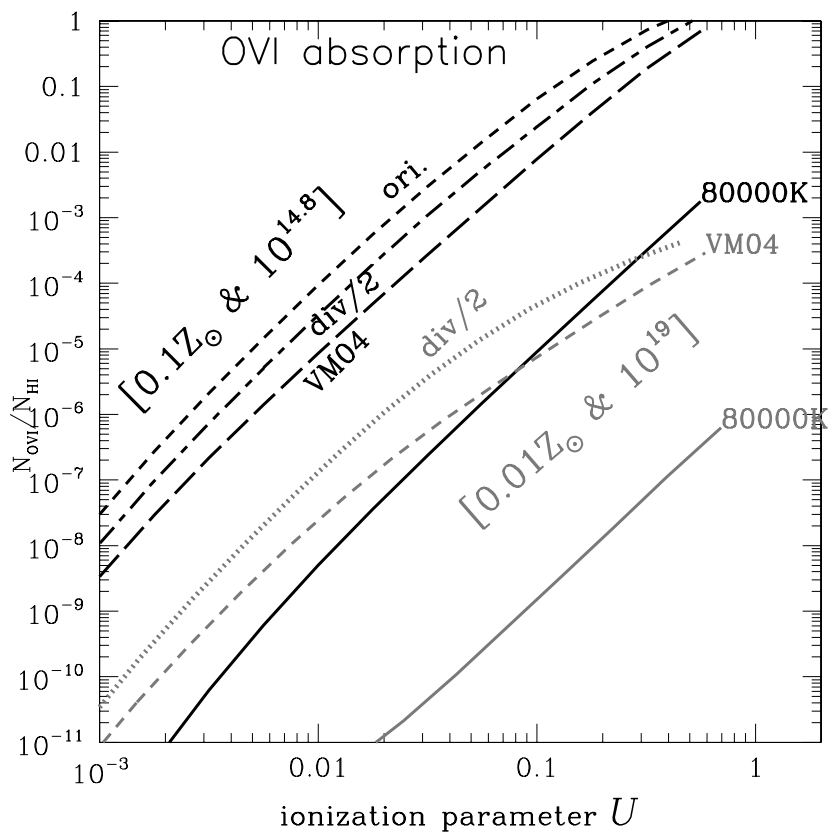

Fig. 9. The column ratio $N_{\mathrm{OVI}} / N_{\mathrm{HI}}$ derived from photoionization by four different SEDs discussed in Sect. 4, as a function of $U$.

calculations for these two resonance lines in Figs. 7 and 8, assuming those SEDs that were most successful in reproducing the observed $N_{\mathrm{CIV}} / N_{\mathrm{HI}}$ ratios. Because both C II and $\mathrm{Mg}$ II are much weaker emission lines than $\operatorname{Ly} \alpha$, we consider it feasible to detect the corresponding absorption doublets only in the case of the thicker $\mathrm{HI}$ absorbers. For instance, for an absorber with $N_{\mathrm{HI}} \simeq 10^{19} \mathrm{~cm}^{-2}$, we expect the C II and Mg II columns to be of the order of $10^{14}$ and $10^{13} \mathrm{~cm}^{-2}$, respectively, assuming a metallicity of $0.01 Z_{\odot}$. Interestingly, the behaviour of the $N_{\mathrm{CII}} / N_{\mathrm{HI}}$ and $N_{\mathrm{MgII}} / N_{\mathrm{HI}}$ ratios is relatively flat in the strong absorber case, with a dependence on $U$ that is much weaker than was the case for C IV. This property would facilitate the determination of the gas metallicity. A possible strategy would be to use Mg II to ascertain the metallicity and then to use the appropriate C IV curve to constrain $U$.

\subsection{Absorption by higher ionization species: O VI 1035 and $N$ V 1240}

McCarthy (1993) produced a composite optical-UV spectrum of 3CR and $1 \mathrm{Jy}$ sources (redshifts up to 3 ) that is useful for estimating typical strengths of various emission lines. Their composite shows that the strongest resonance emission lines in radio galaxies after Ly $\alpha$ and C IV $\lambda \lambda 1549$ are (in order of decreasing flux) O VI $\lambda 1035$, O IV+Si IV $\lambda 1402, \mathrm{~N}$ v $\lambda 1240$, Mg II $\lambda \lambda 2798$, and C II $\lambda \lambda 1335$. Because O IV+Si IV $\lambda 1402$ consists of a blend of two emission doublets, it is unlikely that the corresponding absorption lines could be disentangled. The other resonance lines left to consider are O VI and N V. In Figs. 9 and 10, we present the column ratios $N_{\mathrm{OVI}} / N_{\mathrm{HI}}$ and $N_{\mathrm{NV}} / N_{\mathrm{HI}}$, respectively, as a function of $U$. One can see from these figures that the ionization parameter could be considerably better constrained if data on these resonance lines were obtained. Thus, obtaining highresolution optical spectra over all emission lines is essential for better constraining the properties of these haloes.

Acknowledgements. One of the authors (LB) acknowledges financial support from CONACyT grants J-50296 and 40096-E and the UNAM PAPIIT grants 113002 and 118601 . RJW and MJJ acknowlege the support of PPARC PDRAs. 


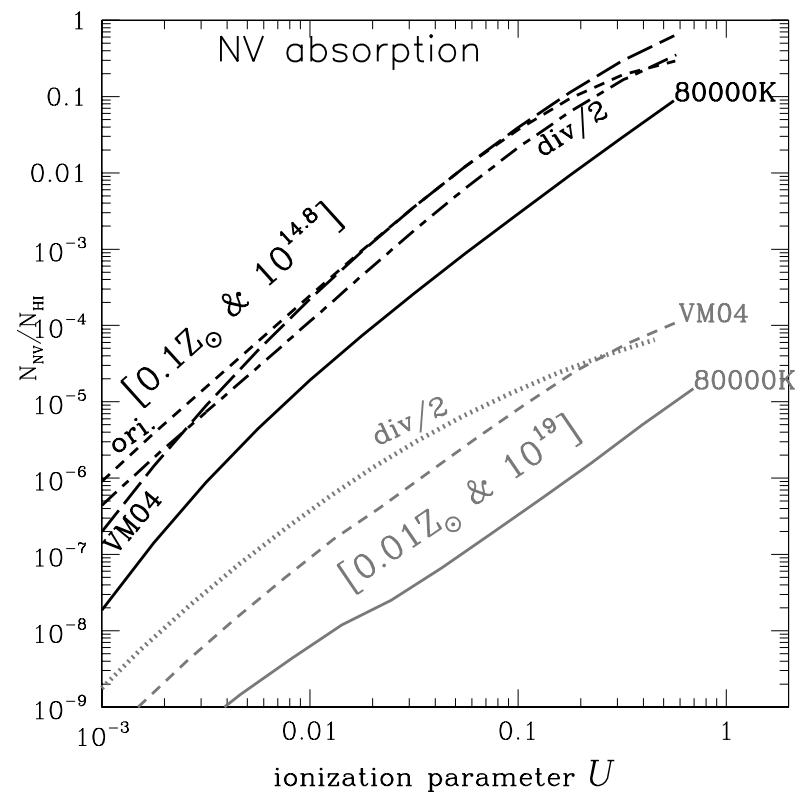

Fig. 10. The column ratio $N_{\mathrm{NV}} / N_{\mathrm{HI}}$ derived from photoionization by four different SEDs discussed in Sect. 4, as a function of $U$.

RAEF is affilliated to the Research and Science Support Department of the European Space Agency. Diethild Starkmeth helped us with proofreading. We acknowledge the technical support of Liliana Hernández and Carmelo Guzmán for configuring the Linux workstation Deneb.

\section{References}

Adelberger, K. L., Shapley, A. E., Steidel, C. C., et al. 2005, ApJ, 629, 636 Anders, E., \& Grevesse, N. 1989, Geochim. Cosmochim. Acta, 53, 197 Antonucci, R. 1993, ARA\&A, 31, 473

Antonucci, R. R. J., Kinney, A. L., \& Ford, H. C. 1989, ApJ, 342, 64 Barthel, P. D. 1989, ApJ, 336, 606

Beckman, J. E., Rozas, M., Zurita, A., Watson, R. A., \& Knapen, J. H. 2000, AJ, 119,2728

Binette, L., Dopita, M. A., \& Tuohy, I. R. 1985, ApJ, 297, 476

Binette, L., Wang, J. C. L., Zuo, L., \& Magris, C. G. 1993, AJ, 105, 797

Binette, L., Kurk, J. D., Villar-Martín, M., \& Röttgering, H. J. A. 2000, A\&A, $356,23(\mathrm{~B} 00)$
Binette, L., Groves, B., Villar-Martín, M., Fosbury, R. A. E., \& Axon, D. J. 2003, A\&A, 405, 975 (BG03)

Cerviño, M., Mas-Hesse, J. M., \& Kunth, D. 2004, available at http://www. laeff.esa.es/users/mcs/SED (CMK04)

Cooke, A. J., Espey, B., \& Carswell, R. F. 1997, MNRAS, 284, 552

Dopita, M. A., \& Sutherland, R. S. 1996, ApJS, 102, 161

Fardal, M. A., Giroux, M. L., \& Shull, J. M. 1998, AJ, 115, 2206 (FGS)

Ferruit, P., Binette, L., Sutherland, R. S., \& Pécontal, E. 1997, A\&A, 322, 73

Fosbury, R. A. E., Villar-Martín, M., Humphrey, A., et al. 2003, ApJ, 596, 797

Giammanco, C., Beckman, J. E., \& Cedrés, B. 2005, A\&A, 438, 599

Haardt, F., \& Madau, P. 1996, ApJ, 461, 20

Haas, M., Siebenmorgen, R., Schulz, B., Krügel, E., \& Chini, R. 2005, A\&A, 442, L39

Heckman, T. M., Miley, G. K., van Breugel, W. J. M., \& Butcher, H. R. 1981, ApJ, 247, 403

Holden, B. P., Stanford, S. A., Rosati, P., et al. 2001, AJ, 122, 629

Jarvis, M. J., Wilman, R. J., Röttgering, H. J. A., \& Binette, L. 2003, MNRAS, 338, 263 (J03)

Jimenez, R., \& Haiman, Z. 2006, Nature, 440, 501

Krause, M. 2002, A\&A, 386, L1

Krause, M. 2005, A\&A, 436, 845

Kriss, G. A., Shull, J. M., Oegerle, W., et al. 2001, Science, 293, 1112

McCarthy, P. J. 1993, ARA\&A, 31, 639

Oke, J. B., \& Korycansky, D. G. 1982, ApJ, 255, 11

Pettini, M., Shapley, A. E., Steidel, C. C., et al. 2001, ApJ, 554, 981

Relaño, M., Peimbert, M., \& Beckman, J. 2002, ApJ, 564, 704

Röttgering, H., \& Miley, G. 1997, in The Early Universe with the VLT (Berlin: Springer), ed. J. Bergeron, 285

Röttgering, H. J. A., Hunstead, R. W., Miley, G. K., van Ojik, R., \& Wieringa, M. H. 1995, MNRAS, 277, 389

Schaerer, D. 2002, A\&A, 382, 28

Shields, G. A., \& Searle, L. 1978, ApJ, 222, 821

Smette, A., Heap, S. R., Williger, G. M., et al. 2002, ApJ, 564, 542

Steidel, C. C., Adelberger, K. L., Shapley, A. E., et al. 2000, ApJ, 532, 170

Telfer, R. C., Kriss, G. A., Zheng, W., Davidsen, A. F., \& Tytler, D. 2002, ApJ, 579,500

van Breukelen, C., Jarvis, M. J., \& Venemans, B. P. 2005, MNRAS, 359, 895

van Ojik, R. 1995, Ph.D. Thesis, University of Leyden, Holland

van Ojik, R., Röttgering, H. J. A., Miley, G. K., \& Hunstead, R. W. 1997, A\&A, 317, 358 (VO97)

van Breugel, W. J. M., Miley, G. K., McCarthy, P. J., \& Spinrad, H. 1997, The Hubble Space Telescope and the High Redshift Universe, 369

Vernet, J., Fosbury, R. A. E., Villar-Martín, M., et al. 2001, A\&A, 366, 7

Villar-Martín, M., Cerviño, M., \& González Delgado, R. M. 2004, MNRAS, 355,1132 (VM04)

Wilman, R., Jarvis, M. J., J., Röttgering, H. J. A., \& Binette, L. 2004, MNRAS, 351, 1109 (W04)

Wilman, R. J., Gerssen, J., Bower, R. G., et al. 2005, Nature, 436, 227

Zurita, A., Beckman, J. E., Rozas, M., \& Ryder, S. 2002, A\&A, 386, 801 